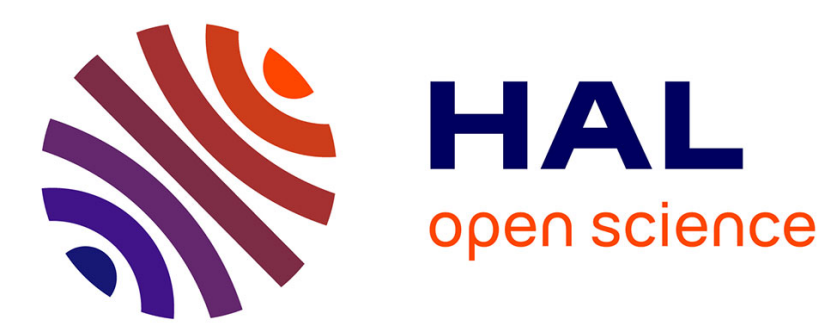

\title{
K-balanced games and capacities
}

Pedro Miranda, Michel Grabisch

\section{To cite this version:}

Pedro Miranda, Michel Grabisch. K-balanced games and capacities. 2008. halshs-00344809

\section{HAL Id: halshs-00344809 \\ https://shs.hal.science/halshs-00344809}

Submitted on 5 Dec 2008

HAL is a multi-disciplinary open access archive for the deposit and dissemination of scientific research documents, whether they are published or not. The documents may come from teaching and research institutions in France or abroad, or from public or private research centers.
L'archive ouverte pluridisciplinaire HAL, est destinée au dépôt et à la diffusion de documents scientifiques de niveau recherche, publiés ou non, émanant des établissements d'enseignement et de recherche français ou étrangers, des laboratoires publics ou privés. 


\section{Documents de Travail du Centre d'Economie de la Sorbonne}

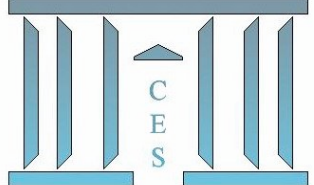

$\boldsymbol{k}$-balanced games and capacities

Pedro MiRANDA, Michel GRABISCH

2008.79 


\title{
$k$-balanced games and capacities
}

\author{
Pedro Miranda* \\ Dept. of Statistics and O.R. \\ Universidad Complutense de Madrid \\ Plaza de Ciencias, 3 \\ 28040 Madrid (Spain) \\ pmiranda@mat.ucm.es \\ Michel Grabisch \\ Centre d'Économie de la Sorbonne \\ Université Paris I-Panthéon-Sorbonne \\ 106-112, Bd. de l'Hôpital \\ 75013 Paris (France) \\ michel.grabisch@univ-paris1.fr
}

\section{Abstract}

In this paper we present a generalization of the concept of balanced game for finite games. Balanced games are those having a nonempty core, and this core is usually considered as the solution of the game. Based on the concept of $k$-additivity, we define the so-called $k$-balanced games and the corresponding generalization of core, the $k$-additive core, whose elements are not directly imputations but $k$-additive games. We show that any game is $k$-balanced for a suitable choice of $k$, so that the corresponding $k$-additive core is not empty. For the games in the $k$ additive core, we propose a sharing procedure to get an imputation and a representative value for the expectations of the players based on the pessimistic criterion. Moreover, we look for necessary and sufficient conditions for a game to be $k$-balanced. For the general case, it is shown

${ }^{*}$ Corresponding author: Pedro Miranda. Dept. of Statistics and O.R. Universidad Complutense de Madrid. Address: Plaza de Ciencias, 3, Ciudad Universitaria, 28040, Madrid (Spain). Tel: (+34) 9139444 19. Fax: (+34) 9139446 06. e-mail: pmiranda@mat.ucm.es 
that any game is either balanced or 2-balanced. Finally, we treat the special case of capacities.

Keywords: Cooperative Games, $k$-additivity, balanced games, capacities, core

\section{Introduction}

One of the main problems of cooperative game theory is to define a solution of a game $\nu$, that is, supposing that all players join the grand coalition $N$, an imputation to each player represents a sharing of the total worth of the game $\nu(N)$. In the case of finite games of $n$ players, an imputation $^{1}$ can be written as a $n$-tuple $\left(x_{1}, \ldots, x_{n}\right)$ such that $\sum_{i=1}^{n} x_{i}=\nu(N)$. Of course, some rationality criterion should prevail when defining the sharing.

In this respect, the core is perhaps the most popular solution of a game. It is defined as the set of imputations $\phi$ on $N$ such that

$$
\sum_{i \in A} x_{i} \geq \nu(A), \forall A \subseteq N, A \neq \emptyset \text { and } \sum_{i=1}^{n} x_{i}=\nu(N)
$$

In this case, $\left(x_{1}, \ldots, x_{n}\right)$ is a possible satisfactory sharing function because no player can do better, even when forming coalitions with other players.

It is a well known fact that the core is nonempty if and only if the game is balanced [1]. However, there are games whose core is empty. It is then necessary to give an alternative solution. In this sense, many possibilities have been proposed in the literature, as stable sets, Shapley index, Banzhaf index, the $\epsilon$-core, the kernel, the nucleolus, etc. (see e.g. [6]).

On the other hand, Grabisch has defined in [9] the concept of $k$-additive capacities (capacities are monotone games). These capacities can be defined with a reduced number of coefficients and they bring up a model that is both flexible and simple to use. For $k$-additive capacities, the value of $k$ (varying between 1 and $n$ ) denotes the maximum cardinality for which the Möbius transform [14] does not vanish, i.e., $k$ represents the maximum cardinality for which subsets can reach importance by their own. The concept of $k$-additivity extends the concept of additivity in the sense that probability measures are indeed 1-additive capacities. Therefore, $k$-additive capacities generalize the concept of probability and they fill the gap between probabilities and

\footnotetext{
${ }^{1}$ The usage in cooperative game theory is to call this a pre-imputation. The distinction being unimportant here since we deal with the core, we keep the name imputation for simplicity.
} 
general capacities. Moreover, as they are defined in terms of the Möbius transform and this transform can be applied to the characteristic function of any game (not necessarily monotone), the concept of $k$-additivity can be extended to games as well.

The aim of this paper is to provide another solution concept, called the $k$-additive core, which we think to be a natural generalization of the core. The basic idea is to remark that an imputation is nothing else than an additive game, and if the core is empty, we may allow to search for games more general than additive ones, namely $k$-additive games, dominating the game. This leads to the concept of $k$-imputation, that is, an imputation over all coalitions of at most $k$ players. We present a generalization of the concept of balanced games, that we will call $k$-balanced games; as it will be explained below, these games are those admitting a dominating $k$-additive game and no dominating $(k-1)$-additive game. We will see that any game is $k$-balanced for a suitable choice of $k$, so that this concept can be applied to any game. By definition of the $k$-additive core, the total worth assigned to a coalition will be always greater or equal than the worth the coalition can achieve by itself, thus keeping the essential spirit of the notion of core; however, the precise sharing among players has still to be decided (e.g., by some sharing or bargaining process) among each group of at most $k$ players.

Moreover, from a $k$-additive imputation, we propose a procedure to obtain a representative value (that in general is not an imputation) for each dominating $k$-additive game based on a pessimistic criterion; for each player, this value represents its minimum payoff. From this, we show that the value of $k$ could be seen as a degree of stability of the game. Hence, players should prefer $k$ as small as possible, which shows that if the core is nonempty, imputations from the core should be taken in priority.

Then, we deal with the problem of deriving a classical imputation from a $k$-additive imputation. In this sense, we propose a new sharing procedure. Anyway, any procedure to derive an imputation could be applied.

Finally, we look for necessary and sufficient conditions for a game to be $k$-balanced for a fixed value of $k$. In particular we show that any game admits a 2-additive game dominating it, so that any game is either balanced or 2-balanced. If we impose monotonicity, that is, if we deal with capacities instead of games, then the situation is more complex. 
The paper is organized as follows: In Section 2 we give the basic concepts on games and capacities that we will need throughout the paper. We also introduce the concept of balanced game and give its interpretation. In Section 3 we introduce the concept of $k$-balanced games; Section 4 is devoted to the deriving possible solutions from the $k$-additive core. In Sections 5 and 6 we look for necessary and sufficient conditions for a given game (resp. capacity) to be $k$-balanced (resp. $k$-balanced monotone). We finish with the conclusions and open problems.

\section{Basic concepts}

Let us consider a set of $n$ players $N=\{1, \ldots, n\}$. Coalitions of players are subsets of $N$, denoted by capital letters $A, B$, and so on, and also $\left\{i_{1}, \ldots, i_{j}\right\}$. Whenever possible, we will omit braces in order to avoid a heavy notation, so $\left\{i_{1}, \ldots, i_{j}\right\}$ turns into $i_{1}, \ldots, i_{j}$, especially for singletons and pairs. The set of all subsets of $N$ is denoted by $\mathcal{P}(N)$, while the set of all subsets of $N$ of cardinality smaller or equal than $k$ is denoted by $\mathcal{P}^{k}(N)$. We start with an example that will play the role of running example for the paper.

Example 1. Three workers share the same office. They need a new bookshelves in order to store their dossiers. However, the number of dossiers increases very quickly so that when the bookshelves arrive, the situation is as follows: The first worker has enough material to fill all the shelves, while the other two workers have enough material to fill $70 \%$ of the space each of them. How should the space be shared?

Definition 1. [13] A game over $N$ is a mapping $\nu: \mathcal{P}(N) \rightarrow \mathbb{R}$ (called characteristic function) satisfying $\nu(\emptyset)=0$.

If, in addition,

(i) $\nu$ satisfies $\nu(A) \leq \nu(B)$ whenever $A \subseteq B$, the game $\nu$ is said to be monotone;

(ii) $\nu$ satisfies $\nu(A \cup B)=\nu(A)+\nu(B)$ whenever $A, B \subseteq N, A \cap B=\emptyset$, the game is said to be additive.

From the point of view of Game Theory, for any $A \subseteq N$, the value $\nu(A)$ represents the minimum asset the coalition of players $A$ will win if the game is played, whatever the remaining players may do, i.e., $\nu(A)$ is the payoff that coalition $A$ can guarantee for itself. 
Definition 2. A non-additive measure [5] or capacity [3] or fuzzy measure [18] $\nu$ over $N$ is a monotone game with $\nu(N)=1$.

Consider a monotone game different from the trivial game defined by $\nu(A)=0, \forall A \subseteq N$. In this case, we can divide all the values of $\nu$ by $\nu(N)$ so that we obtain a new game $\nu^{\prime}$ equivalent to $\nu$. Then, $\nu^{\prime}$ is a capacity and we conclude that any monotone game can be equivalently represented by a capacity. From now on, we will denote by $\mathcal{G}(N)$ the set of all games over $N$ and by $\mathcal{F} \mathcal{M}(N)$ the set of all capacities.

Example 1. (Continued) The situation stated previously can be modelled through the capacity

$$
\nu(1)=1, \nu(2)=\nu(3)=0.7, \nu(i, j)=1, \forall i, j, \nu(1,2,3)=1 .
$$

There are other set functions that can be used to equivalently represent a game. We will need in this paper the so-called Möbius transform.

Definition 3. [14, 12] Let $\nu$ be a game on $N$. The Möbius transform (or dividends) of $\nu$ is the function defined on $\mathcal{P}(N)$ by

$$
m(A):=\sum_{B \subseteq A}(-1)^{|A \backslash B|} \nu(B), \forall A \subseteq N .
$$

The Möbius transform given, the original characteristic function can be recovered through the Zeta transform [2]:

$$
\nu(A)=\sum_{B \subseteq A} m(B) .
$$

The value $m(A)$ represents the strength of subset $A$ in any coalition in which it appears. Given a Möbius inverse, necessary and sufficient conditions for its Zeta transform to be a capacity have been investigated. The following can be proved:

Proposition 1. [2] A set of $2^{n}$ coefficients $m(A), A \subseteq N$, corresponds to the Möbius transform of a capacity if and only if

(i) $m(\emptyset)=0, \sum_{A \subseteq N} m(A)=1$,

(ii) $\sum_{i \in B \subseteq A} m(B) \geq 0$, for all $A \subseteq N, \forall i \in A$. 
Remark that in general $m(A)$ can attain negative values. If we restrict to the case for which the Möbius transform can attain only nonnegative values, we obtain a special subfamily of capacities called belief functions. Belief functions come from the Theory of Evidence developed by Dempster [4] and Shafer [15].

Definition 4. Let $\nu, \nu^{*} \in \mathcal{G}(N)$. We say that $\nu^{*}$ dominates $\nu$, denoted by $\nu^{*} \geq \nu$, if

$$
\nu^{*}(A) \geq \nu(A), \quad \forall A \subseteq N, \text { and } \nu^{*}(N)=\nu(N)
$$

Let us now recall the basic results on balanced games.

Definition 5. Let $\nu \in \mathcal{G}(N)$. We say that a vector $: x=\left(x_{1}, \ldots, x_{n}\right) \in \mathbb{R}^{n}$ is an imputation for $\nu$ if it satisfies

$$
\sum_{i=1}^{n} x_{i}=\nu(N)
$$

Remark 1. For any $x \in \mathbb{R}^{n}$, it is convenient to use the notation $x(A):=\sum_{i \in A} x_{i}$, for all $A \subseteq N$, with the convention $x(\emptyset)=0$. Thus, $x$ identifies with an additive game.

The value $x_{i}$ is the asset player $i$ receives when sharing $\nu(N)$. Several solutions for obtaining imputations have been proposed, the Shapley value [16] being among the most popular. However, it could be the case that some of the players do not consider their corresponding value as a satisfactory asset. Suppose that the imputation satisfies $x(A) \geq \nu(A)$, for all $A \subseteq N$. Then, no subcoalition of players has interest to form, since they will receive more by the sharing $\left(x_{1}, \ldots, x_{n}\right)$. In other words, any such $\left(x_{1}, \ldots, x_{n}\right)$ is a possible satisfactory imputation for all players.

Definition 6. [17] Let $\nu$ be a game. The core of $\nu$, denoted by $\mathcal{C}(\nu)$, is defined by

$$
\mathcal{C}(\nu):=\left\{x \in \mathbb{R}^{n} \mid x(A) \geq \nu(A), \forall A \subseteq N, x(N)=\nu(N)\right\}
$$

Remark 2. Since by Remark 1 any $x \in \mathbb{R}^{n}$ induces an additive game, the core can be equivalently defined as the set of additive games dominating $\nu$.

When the core is nonempty, it is usually taken as the solution of the game. However, there are games with an empty core. Then, the following definition arises: 
Definition 7. [13] A game $\nu \in \mathcal{G}(N)$ is balanced if $\mathcal{C}(\nu) \neq \emptyset$.

For the special case of $\nu$ being a capacity, if $\left(x_{1}, \ldots, x_{n}\right)$ is in the core, it follows that $\left(x_{1}, \ldots, x_{n}\right)$ determines a probability distribution on $N$ dominating $\nu$. Thus, in this case, $\mathcal{C}(\nu)$ coincides with the set of all probabilities dominating $\nu$, and the concept of balanced games translates to capacities as follows:

Definition 8. A capacity $\nu$ is said to be balanced if it admits a dominating probability measure.

Note that for the case of the core, giving a dominating additive game, the value $x_{i}$ coincides with $m(i)$.

Let us turn to the concept of $k$-additivity. In order to define a capacity, $2^{n}-2$ values are necessary. The number of coefficients grows exponentially with $n$, and so does the complexity of the problem. This drawback reduces considerably the practical use of capacities. Then, some subfamilies of capacities have been defined in an attempt to reduce complexity. In this paper we will use $k$-additive capacities. This choice is based in two facts: First, as we will see below, $k$ additive capacities are a generalization of probability distributions and thus, they are suitable to define a concept extending the core. Secondly, they are defined in terms of the Möbius transform and therefore, it seems natural to use this transformation in the sharing procedure. Moreover, the value of $k$ can be interpreted as the maximum size for which a coalition has a non-null dividend and consequently, the maximum cardinality for which a sharing is needed; this will be interesting when interpreting $k$-balanced games.

Definition 9. [8] A game $\nu$ is said to be $k$-order additive or $k$-additive for short if its Möbius transform vanishes for any $A \subseteq N$ such that $|A|>k$, and there exists at least one subset $A$ of exactly $k$ elements such that $m(A) \neq 0$.

Additive games are 1-additive games, and so probability measures are 1-additive capacities (indeed, 1-additive belief functions); thus, $k$-additive capacities generalize probability measures. More about $k$-additive capacities can be found, e.g., in [10].

The value $k$ represents the maximum cardinality for which subsets can reach importance by their own. We will denote by $\mathcal{G}^{k}(N)\left(\right.$ resp. $\left.\mathcal{F} \mathcal{M}^{k}(N)\right)$ the set of games (resp. capacities) being $k^{\prime}$-additive, for $1 \leq k^{\prime} \leq k$. 


\section{$3 \quad k$-balanced games}

Let $\nu$ be a game. If the core of $\nu$ is empty, it is not possible to find a satisfactory imputation for all players (in terms of coalitional stability; there are other solution concepts for a game that are appealing imputations, as the nucleolus or the Shapley value to cite a few). Since from Remark 2 the core is the set of 1-additive dominating games, when it is empty it is natural to look for 2-additive games dominating $\nu$; if this set is empty too, then look for 3 -additive games dominating $\nu$ and so on. This leads us to the following definition.

Definition 10. A game $\nu$ on $N$ is called $k$-balanced if there exists a $k$-additive game dominating it and no game in $\mathcal{G}^{k-1}(N)$ dominates $\nu$.

In this sense, a balanced game is indeed a 1-balanced game. We will denote by $\mathcal{B A L}^{k}(N)$ the set of all $k$-balanced games on $N$. As any game $\nu$ is $k$-additive for a suitable choice of $k$, then $\nu$ is $k^{\prime}$-balanced, for some value $k^{\prime} \leq k$. This implies that the collection $\mathcal{B A L}^{k}(N), k=1, \ldots, n$ determines a partition of $\mathcal{G}(N)$ :

$$
\mathcal{B} \mathcal{A} \mathcal{L}^{1}(N) \cup \cdots \cup \mathcal{B} \mathcal{A} \mathcal{L}^{n}(N)=\mathcal{G}(N), \quad \mathcal{B A L}^{i}(N) \cap \mathcal{B A L}^{j}(N)=\emptyset, i \neq j .
$$

We will see in Section 5 that in fact this structure simplifies drastically.

Definition 11. (i) Given a game $\nu$, we define the $k$-additive core of $\nu$, denoted by $\mathcal{C}^{k}(\nu)$, as the set of all $k$-additive games dominating $\nu$, i.e.,

$$
\mathcal{C}^{k}(\nu):=\left\{\nu^{*} \in \mathcal{G}^{k}(N) \mid \nu^{*}(A) \geq \nu(A), \forall A \subset N, \nu^{*}(N)=\nu(N)\right\} .
$$

(ii) A $k$-imputation for $\nu$ is a sharing of the worth $\nu(N)$ among all coalitions of at most $k$ players, i.e., it is a vector $x \in \mathbb{R}^{\left|\mathcal{P}^{k}(N)\right|-1}$ satisfying $\sum_{A \in \mathcal{P}^{k}(N), A \neq \emptyset} x_{A}=\nu(N)$. Any $\nu^{*} \in \mathcal{C}^{k}(\nu)$ defines a $k$-imputation, which is simply its Möbius transform $m^{*}$, i.e., $x_{A}:=$ $m^{*}(A)$, for all $A$ in $\mathcal{P}^{k}(N) \backslash\{\emptyset\}$.

We stress the fact that any $k$-order imputation $x$ in the $k$-additive core of a game $\nu$ is such that the total amount given to coalition $A$, which is $\nu^{*}(A):=\sum_{B \subseteq A \mid B \in \mathcal{P}^{k}(X)} x(B)$, is at least equal to $\nu(A)$. This is our generalization of coalitional stability. 
As stated before, from a mathematical point of view the set of capacities constitutes a special class of games. In many applications (for example in Imprecise Probabilities [19]), it is interesting to study the set of probabilities dominating a given capacity; in these frameworks, it makes no sense to remove the condition of monotonicity. Then, it seems interesting to extend the concept of dominance to the $k$-additive case, but keeping monotonicity. This leads us to the following concept:

Definition 12. A capacity $\nu$ on $N$ is $k$-balanced monotone if there exists a $k$-additive capacity dominating it and no capacity in $\mathcal{F} \mathcal{M}^{k-1}(N)$ dominates $\nu$.

We will denote the set of all $k$-balanced monotone capacities by $\mathcal{B A L}_{\mathcal{M}}^{k}(N)$.

Definition 13. Given a $k$-balanced monotone capacity $\nu$, we define the $k$-additive monotone core of $\nu$, denoted by $\mathcal{C}_{\mathcal{M}}^{k}(\nu)$, as the set of all $k$-additive capacities dominating $\nu$. Any $\phi \in \mathcal{C}_{\mathcal{M}}^{k}(\nu)$ defines a $k$-imputation, which is simply its Möbius transform.

As for the general case, $\mathcal{B} \mathcal{A} \mathcal{L}_{\mathcal{M}}^{k}(N), k=1, \ldots, n$, forms a partition of $\mathcal{F} \mathcal{M}(N)$, i.e.,

$$
\mathcal{B A}_{\mathcal{L}}^{1}{ }_{\mathcal{M}}(N) \cup \cdots \cup \mathcal{B} \mathcal{A} \mathcal{L}_{\mathcal{M}}^{n}(N)=\mathcal{F} \mathcal{M}(N), \quad \mathcal{B A} \mathcal{L}_{\mathcal{M}}^{i}(N) \cap \mathcal{B} \mathcal{A L}_{\mathcal{M}}^{j}(N)=\emptyset, i \neq j
$$

For the special case of belief functions, the following can be shown:

Proposition 2. A necessary and sufficient condition for a capacity $\nu$ to have a dominating belief function is $\mathcal{C}_{\mathcal{M}}^{1}(\nu) \neq \emptyset$.

Proof: If $\nu \in \mathcal{B A}_{\mathcal{M}}^{1}(N)$, then it admits a dominating probability measure, and thus, a dominating belief function.

Conversely, suppose that $\nu$ admits a dominating belief function $\nu^{*}$, with corresponding Möbius transform $m^{*}$. Consider an order $i_{1} \preceq i_{2} \preceq \cdots \preceq i_{n}$ on $N$ and let us define $P$ by:

$$
P\left(i_{j}\right):=\sum_{A \mid i_{j} \in A \subseteq\left\{i_{1}, \ldots, i_{j}\right\}} m^{*}(A) .
$$

It is straightforward to see that $P$ is a probability measure dominating $\nu^{*}$, whence the result.

As a consequence of this result, the following can be proved for $k$-balanced capacities $(k>1)$ : 
Corollary 1. If $\nu$ is a capacity such that $\nu \in \mathcal{B A}_{\mathcal{M}}^{k}(N), k \geq 2$, then for any capacity $\nu^{*} \in$ $\mathcal{C}_{\mathcal{M}}^{k}(\nu)$, there is at least one subset $A \in \mathcal{P}^{k}(N)$ such that $m^{*}(A)<0$.

This means that if a capacity has an empty core, we necessarily need to allow negative dividends in order to dominate it.

Finally, note that if $\nu$ is a capacity, we can look for the values $k$ and $k^{\prime}$ such that $\nu \in \mathcal{B A L}^{k}(N)$ and $\nu \in \mathcal{B A}_{\mathcal{M}}^{k^{\prime}}(N)$. Obviously $k \leq k^{\prime}$ holds in general, and next example shows a case where $k<k^{\prime}$.

Example 1. (Continued) For the game $\nu$ modelling the problem of sharing the bookshelves, it can be seen that no additive game can dominate $\nu$. Thus, this game is not balanced. On the other hand, the game $\nu^{*}$ whose Möbius transform is given by $m^{*}(i)=2, m^{*}(i, j)=-\frac{5}{3}, m^{*}(N)=0$, dominates $\nu$ as next table shows:

\begin{tabular}{|c|lllrrrc|}
\hline & $\{1\}$ & $\{2\}$ & $\{3\}$ & $\{1,2\}$ & $\{1,3\}$ & $\{2,3\}$ & $\{N\}$ \\
\hline$m^{*}$ & 2 & 2 & 2 & $-\frac{5}{3}$ & $-\frac{5}{3}$ & $-\frac{5}{3}$ & 0 \\
\hline$\nu^{*}$ & 2 & 2 & 2 & $\frac{7}{3}$ & $\frac{7}{3}$ & $\frac{7}{3}$ & 1 \\
\hline$\nu$ & 1 & 0.7 & 0.7 & 1 & 1 & 1 & 1 \\
\hline
\end{tabular}

Thus, $\nu \in \mathcal{B A L}^{2}(N)$.

Moreover, there is no 2-additive capacity $\nu^{*}$ dominating $\nu$; for if such $\nu^{*}$ exists, from the definition of dominance and by monotonicity, it would follow that $\nu^{*}(1) \geq \nu(1)=1$ and $\nu^{*}(N)=$ $1 \geq \nu^{*}(1) ;$ therefore, $\nu^{*}(1)=1=m^{*}(1)$. On the other hand, $\nu^{*}(1,2)=1$, but $1 \geq \nu^{*}(1,2)=$ $m^{*}(1)+m^{*}(2)+m^{*}(1,2)$, whence $m^{*}(1,2)=-m^{*}(2)$; the same can be done for $\{1,3\}$, whence $m^{*}(1,3)=-m^{*}(3)$.

Finally, $1=\nu^{*}(2,3)=m^{*}(2)+m^{*}(3)+m^{*}(2,3)$, but as $m^{*}(2)=\nu^{*}(2) \geq 0.7$ and $m^{*}(3)=$ $\nu^{*}(3) \geq 0.7$, we conclude that $m^{*}(2,3) \leq-0.4$. As $\sum_{A \subseteq N} m^{*}(A)=1$, we necessarily need $m^{*}(N)>0$. Consequently, $\nu \notin \mathcal{B} \mathcal{A} \mathcal{L}_{M}^{2}(N)$ and this implies that $\nu \in \mathcal{B A L}_{M}^{3}(N)$, as $\nu$ is itself a 3-additive monotone game. 


\section{Building a solution from the $k$-additive core}

\subsection{Defining an imputation from a $k$-imputation}

Suppose $\nu$ is a $k$-balanced game, and take any $\nu^{*} \in \mathcal{C}^{k}(\nu)$. We know from Definition 11 that the Möbius transform $m^{*}$ of $\nu^{*}$ defines a $k$-imputation. Since a $k$-imputation is not an individual allocation to players (i.e., a solution of the game), we have to derive somehow an imputation from $m^{*}$. A natural way is to proceed as follows. For any $A \in \mathcal{P}^{k}(N)$ such that $m^{*}(A) \neq 0$ :

- If $m^{*}(A)>0$, the value $m^{*}(A)$ is shared among players in coalition $A$ in some way. In this case, all players in coalition $A$ receive a nonnegative quantity.

- If $m^{*}(A)<0$, the payment of value $-m^{*}(A)$ must be shared among players in coalition $A$ in some way. In this case, all players in coalition $A$ pay a nonnegative quantity.

Indeed, following the interpretation of Möbius transform, we are sharing the dividend of the coalition among its members. Remark that when sharing $m^{*}(A)$, we do not allow that some of the players receive a positive quantity and others a negative value. Following this procedure, we derive an imputation in the classical sense.

In the sequel, we propose two easy ways to derive an imputation from a $k$-imputation $m^{*}$. The

first one is to give to each player in $A$ the quantity $\frac{m^{*}(A)}{|A|}$, and we proceed in this way for any $A \subseteq N$. Then, it is well known that doing so we get the Shapley value of $\nu^{*}$.

Let us introduce another sharing procedure, which we call the proportional sharing. For any $A$ such that $m^{*}(A) \neq 0$, the idea is to allocate to player $i \in A$ the value $m^{*}(A)$ in proportion of $m^{*}(i) / \sum_{j \in A} m^{*}(j)$. Specifically, supposing for the moment that $m^{*}(i) \geq 0$ for all $i \in N$ (which is always true if $\nu^{*}$ is monotone):

(i) For any $A \in \mathcal{P}^{k}(N)$ such that $m^{*}(A) \neq 0$, each player $i \in A$ receives the amount

$$
x_{i}(A):=\frac{m^{*}(i)}{\sum_{j \in A} m^{*}(j)} m^{*}(A),
$$

if $\sum_{j \in A} m^{*}(j) \neq 0$, otherwise $x_{i}(A):=\frac{m^{*}(A)}{|A|}$. 
(ii) The imputation $x \in \mathbb{R}^{n}$ derived from $m^{*}$ is defined for player $i$ by

$$
x_{i}:=\sum_{A \in \mathcal{P}^{k}(N), A \ni i} x_{i}(A)
$$

Clearly, $x$ is a well-defined imputation. In a sense, the values $m^{*}(i)$ could be seen as a kind of decisional power to conduct subsequent sharings for all coalitions in $\mathcal{P}^{*}(N)$ containing $i$.

If $m^{*}(i)<0$ for some $i \in N$, the above procedure may lead to counterintuitive results. Indeed, take $A \in \mathcal{P}^{k}(N)$ such that $m^{*}(A)>0$ and $m^{*}(i)<0$ for all $i \in A$. Then the player $i$ who has the lowest value for $m^{*}(i)$ would receive the biggest part of $m^{*}(A)$. To avoid this, we could proceed as follows.

Consider $r:=\min _{i \in N} m^{*}(i)<0$. We define $\nu^{\prime}$ as

$$
\nu^{\prime}(A):=\nu^{*}(A)-r, \forall A \subseteq N
$$

Then, if $m^{\prime}$ denotes the Möbius transform of $\nu^{\prime}$, it follows that $m^{\prime}(i) \geq 0, \forall i \in N$, whence we can apply the proportional sharing. Once the procedure is applied, we subtract $\frac{r}{n}$ from the values of all players in order to get an imputation.

The following lemma gives a justification of the proportional sharing.

Lemma 1. Let $\nu \in \mathcal{G}(N)$. Then there exists $\nu^{*} \in \mathcal{C}^{n}(\nu)$ such that $m^{*}(A)=0, \forall A \subsetneq N,|A|>1$.

Proof: We consider

$$
m^{*}(i):=\max _{i \in A \subseteq\{1, \ldots, i\}}\left\{\nu(A)-\sum_{j \in A, j \neq i} m^{*}(j)\right\}, \forall i \in N .
$$

Finally, we define $m^{*}(N):=\nu(N)-\sum_{i \in N} m^{*}(i)$; this is necessary in order to have $\nu^{*}(N)=\nu(N)$. It is straightforward to see that the game $\nu^{*}$ whose Möbius transform is $m^{*}$ dominates $\nu$; for if we consider $A \subseteq N$, if $A=N$, the result holds by construction; otherwise, let $i_{A}:=\max _{i \in A} i$. Then,

$$
m^{*}\left(i_{A}\right) \geq \nu(A)-\sum_{j \in A, j \neq i_{A}} m^{*}(j) \Leftrightarrow \nu^{*}(A)=\sum_{i \in A} m^{*}(i) \geq \nu(A) .
$$

Therefore, the result holds. 
Remark that for $\nu^{*}$ we only have to share $m^{*}(N)$ among all the players in $N$ to define an imputation for $\nu$. Now, if $\nu^{*}$ satisfies the condition of Lemma 1, then any $\nu^{\prime}$ defined through its Möbius transform by

$$
m^{\prime}(i):=\lambda m^{*}(i), \quad \forall i \in N, \quad m^{\prime}(N)=\nu(N)-\sum_{i \in N} m^{\prime}(i),
$$

satisfies it as well for any $\lambda>1$. In a sense, $\nu^{*}$ and $\nu^{\prime}$ are essentially equivalent (they have the same structure for the Möbius transform) and should lead to the same imputation.

For $\nu^{*}$, let us denote by $\alpha_{i} \in[0,1]$ the proportion of $m^{*}(N)$ that is given to player $i$. Then, the allocation for $i$ is

$$
x_{i}^{*}=m^{*}(i)+\alpha_{i} m^{*}(N) .
$$

As $\nu^{\prime}$ is essentially the same game, the proportion of $m^{\prime}(N)$ that is given to $i$ should be again $\alpha_{i}$. Then, the final value for $i$ is

$$
x_{i}^{\prime}=m^{\prime}(i)+\alpha_{i} m^{\prime}(N) .
$$

On the other hand, note that

$$
m^{\prime}(N)=\nu(N)-\lambda \sum_{i \in N} m^{*}(i)
$$

Then, as $x_{i}^{*}=x_{i}^{\prime}$,

$$
m^{*}(i)+\alpha_{i}\left(\nu(N)-\sum_{i \in N} m^{*}(i)\right)=\lambda m^{*}(i)+\alpha_{i}\left(\nu(N)-\lambda \sum_{i \in N} m^{*}(i)\right)
$$

whence, assuming $\sum_{i \in N} m^{*}(i) \neq 0$,

$$
\alpha_{i}=\frac{(1-\lambda) m^{*}(i)}{(1-\lambda) \sum_{i \in N} m^{*}(i)}=\frac{m^{*}(i)}{\sum_{i \in N} m^{*}(i)}
$$

Example 1. (Continued) In the running example, we have already proved that $\nu \in \mathcal{B A}_{\mathcal{M}}^{3}(N)$ and that indeed $\nu \in \mathcal{C}_{\mathcal{M}}^{3}(\nu)$. The Möbius transform of $\nu$ is given by

\begin{tabular}{|c|ccccccc|}
\hline & $\{1\}$ & $\{2\}$ & $\{3\}$ & $\{1,2\}$ & $\{1,3\}$ & $\{2,3\}$ & $N$ \\
\hline$\nu$ & 1 & 0.7 & 0.7 & 1 & 1 & 1 & 1 \\
\hline$m$ & 1 & 0.7 & 0.7 & -0.7 & -0.7 & -0.4 & 0.4 \\
\hline
\end{tabular}


Let us obtain a possible solution for this game. If we apply the previous procedure, we obtain the imputation $(0.35,0.325,0.325)$. If we consider the Shapley value as solution, we obtain $(0.433,0.283,0.283)$. In this sense, the proportional sharing procedure forces the players with highest values $m^{*}(i)$ to pay more for negative values of $m^{*}(A)$ and offers them a better value if $m^{*}(A)>0$. Then, for this concrete example, as $m^{*}(A)<0$ for all pairs, it provides a more egalitarian solution.

\subsection{Why the $k$-additive core with smallest $k$ should be preferred}

A central question is the following: Why a dominating additive game should be preferred to a dominating 2-additive game? In other words, we need to justify the fact that, for a game $\nu$, we look for a dominating $k$-additive (monotone) game $\nu^{*}$ with $k$ as small as possible. This is important in the sense that we want our solution to be a generalization of the core and not an alternative to it; thus, it is necessary to show that whenever the core is not empty, it still constitutes the solution of the game.

Remark that, depending on the sharing procedure that is applied, the payoffs of the players can vary. If player $i$ is pessimist, he might think that he will receive all the quantities coming from negative Möbius coefficients $m^{*}(A), i \in A$, and nothing at all from the positive Möbius coefficients $m^{*}(A), i \in A$. This provides the minimum value that player $i$ can receive:

Definition 14. Let $\nu$ be a game, and consider $\nu^{*} \in \mathcal{C}^{k}(\nu)$ with Möbius transform $m^{*}$. We define the minimal value for player $i$ as

$$
\underline{x}_{i}^{*}:=m^{*}(i)+\sum_{i \in A, m^{*}(A)<0} m^{*}(A)
$$

We will refer to $\left(\underline{x}_{1}^{*}, \ldots, \underline{x}_{n}^{*}\right)$ as the minimal vector associated to $\nu^{*}$.

These minimal values generalize the corresponding sharing function for balanced games (but are not in general an imputation). At this point, remark that we could have considered other different representative values that also generalize the sharing procedure of balanced games instead of the pessimistic criterion, as for example the optimistic criterion, an adaptation of the Hurwicz criterion, and so on. However, the pessimistic criterion seems to be in our opinion the 
most appropriate, in the sense that it is more conservative. We will come again to this point below. Following this pessimistic behavior, let us show that players should prefer a $k$-additive game with $k$ being as small as possible.

Let us then suppose that a game $\nu$ is dominated by a game $\nu^{*}$. From $\nu^{*}$ it is possible to derive the corresponding minimal values for all players through Eq. (1). In the minimal values we are supposing that we have no information about how $m^{*}(B)$ should be shared (or afforded) among players in coalition $B$; however, it could be the case that we had the additional information that part of $m(B)$, say $r$, must be shared or afforded by a subcoalition of players in $B$, say $A$. Then, we have to consider this new situation when computing the minimal values. Suppose w.l.o.g. that $m^{*}(B)>0$; the natural way to deal with this additional constraint is to add $r$ to $m^{*}(A)$ and skip it from $m^{*}(B)$. This leads us to the following concept.

Definition 15. Let $\nu$ be a game on $N$ with Möbius transform $m$. We say that $\nu^{*} \in \mathcal{G}(N)$ is a contraction of $\nu$ if the Möbius transform $m^{*}$ of $\nu^{*}$ can be put under the following form:

$$
m^{*}(A)=\sum_{B \supseteq A} \lambda(B, A) m(B), \forall A \in \mathcal{P}(N),
$$

where function $\lambda: \mathcal{P}(N) \times \mathcal{P}(N) \rightarrow[0,1]$ is a sharing function such that

$$
\sum_{A \subseteq B} \lambda(B, A)=1, \forall B \subseteq N, \lambda(B, A)=0, \forall A \in \mathcal{P}(N), A \nsubseteq B
$$

If $\nu^{*}$ is a contraction of $\nu$, then $\nu^{*}$ gives more importance (in terms of the Möbius transform) to smaller coalitions than the original $\nu$. Remark that if $\nu$ is a $k$-additive game, then $\nu^{*}$ is at most $k$-additive. Note also that, as $\lambda$ takes values in [0,1], when multiplying it by $m(B)$, if $m(B)>0$ (resp. $m(B)<0$ ), no $A \subseteq B$ can receive a negative (resp. positive) payoff. The Shapley value is just an additive contraction of $\nu$ and similarly, the value derived from the procedure we developed previously for a game $\nu^{*}$ in $\mathcal{C}^{k}(\nu)$ is an additive contraction of $\nu^{*}$.

Proposition 3. Let $\nu$ be a game and consider $\nu^{*} \geq \nu$ with Möbius transform $m^{*}$. Suppose $\nu^{\prime}$ is a contraction of $\nu^{*}$ such that $\nu^{\prime} \geq \nu$. Then, denoting by $\underline{x}_{i}^{\prime}$ the minimal value for player $i$ for $\nu^{\prime}$ and by $\underline{x}_{i}^{*}$ the corresponding value for $\nu^{*}$, we have $\underline{x}_{i}^{\prime} \geq \underline{x}_{i}^{*}, \forall i \in N$.

Proof: Take $i \in N$ and consider $m^{*}(B) \neq 0$. Take $A \subseteq B$. Then, applying Eq. (1), we have the following: 
- If $i \notin B$, then $\underline{x}_{i}^{\prime}=\underline{x}_{i}^{*}$.

- If $i \in B, m^{*}(B)>0$ and $|A|>1$, then $\underline{x}_{i}^{\prime}=\underline{x}_{i}^{*}, \forall i \in B$. If $A=\{j\}$, then $\underline{x}_{i}^{\prime}=\underline{x}_{i}^{*}, \forall i \neq j$ and $\underline{x}_{j}^{\prime}=\underline{x}_{j}^{*}+\lambda(B,\{j\}) m^{*}(B) \geq \underline{x}_{j}^{*}$.

- If $i \in B$ and $m^{*}(B)<0$, then $\underline{x}_{i}^{\prime}=\underline{x}_{i}^{*}, \forall i \in A$ and $\underline{x}_{i}^{\prime}=\underline{x}_{i}^{*}-\lambda(B, A) m^{*}(B) \geq \underline{x}_{i}^{*}, \forall i \in B \backslash A$.

Therefore, $\underline{x}_{i}^{\prime} \geq \underline{x}_{i}^{*}, \forall i \in N$. Following this process for all $B$ and all $A \subseteq B$, the result holds.

In particular, Proposition 3 shows that if a $k$-additive game $\nu^{*}$ dominates $\nu$, but it is also possible to obtain from contraction a $k^{\prime}$-additive dominating game $\nu^{\prime}$ with $k^{\prime}<k$, then all the players should be indifferent or prefer $\nu^{\prime}$ instead of $\nu^{*}$ in terms of their minimal values.

This shows that $k$ might be seen as a measure of stability, in the sense that the smaller $k$ is, the happier the players are and the corresponding game is more "stable". From an interpretational point of view, the players should prefer $k$ as small as possible, as large coalitions would lead to more conflicts to share (or afford) the value $m^{*}(A)$.

If we had considered the optimistic criterion, then the representative value for player $i$ would be given by

$$
\sum_{i \in A, m(A) \geq 0} m(A) .
$$

In this case, players would look for coalitions as large as possible because they are confident about the sharing procedure. This would imply that for balanced games, the core might not provide optimal sharing functions. Indeed, from the point of view of expectations, these games would be quite unstable, as players could feel upset when their expected values could not be attained for all of them.

Suppose that we are dealing with the monotone case and assume that $\nu^{*}$ is a $k$-additive belief function. It can be easily seen that any $(k-1)$-additive belief function $\nu^{\prime}$ obtained by contracting $\nu^{*}$ is also a belief function and $\nu^{\prime} \geq \nu^{*}$, so the choice of $\nu^{\prime}$ instead of $\nu^{*}$ seems rather sensible. For the optimistic criterion, a belief function would be preferred to a probability, that does not seem natural, as the probability dominates the belief function and is simpler. This is another argument in favor of the pessimistic behavior and against the optimistic. 
Note that the result of Proposition 3 only applies when $\nu^{\prime}$ is a contraction of $\nu^{*}$. If this is not the case, we can obtain that some players would prefer $\nu^{*}$. Indeed, it is possible that some players would prefer $\nu^{*} \in \mathcal{C}^{k}(\nu)$ to any other dominating game in $\mathcal{C}^{k^{\prime}}(\nu)$, with $k^{\prime}<k$.

Example 2. Consider $|N|=3$ and the capacity defined by

\begin{tabular}{|c|ccccccc|}
\hline & 1 & 2 & 3 & 1,2 & 1,3 & 2,3 & $1,2,3$ \\
\hline$\nu$ & 0.3 & 0.4 & 0.3 & 0.6 & 0.6 & 0.6 & 1 \\
\hline
\end{tabular}

Let us define the 2-additive game $\nu^{*}$ whose Möbius transform is given by

$$
m^{*}(1)=0.4, m^{*}(2)=0.4, m^{*}(3)=0.3, m^{*}(2,3)=-0.1, m^{*}(A)=0 \text { otherwise }
$$

It is easy to check that $\nu^{*} \geq \nu$ :

\begin{tabular}{|c|ccccccc|}
\hline & 1 & 2 & 3 & 1,2 & 1,3 & 2,3 & $1,2,3$ \\
\hline$\nu^{*}$ & 0.4 & 0.4 & 0.3 & 0.8 & 0.7 & 0.6 & 1 \\
\hline
\end{tabular}

However, it is not possible to derive a 1-additive game dominating $\nu$ from $\nu^{*}$. For if we share $m^{*}(2,3)$ between $\{2\}$ and $\{3\}$, we lose dominance on singletons.

On the other hand, the probability distribution defined by $P(1)=0.3, P(2)=0.4, P(3)=0.3$ dominates $\nu$ and it is the only probability distribution in these conditions.

The minimal values for $\nu^{*}$ are given by $\underline{x}_{1}^{*}=0.4, \underline{x}_{2}^{*}=0.3, \underline{x}_{3}^{*}=0.2$. Thus, player 1 would prefer to obtain his sharing from $\nu^{*}$ instead of the sharing given by the probability. However, the other players would prefer the payoffs from the probability. Then, $\nu^{\prime}$ and $\nu^{*}$ are not comparable.

\section{$5 \quad k$-balanced families}

We turn to the problem of finding necessary and sufficient conditions for the nonemptiness of the $k$-additive core.

Consider a game $\nu \in \mathcal{G}(N)$, and the following linear program with variables $m(B), B \in \mathcal{P}^{k}(N)$, which we will call (P1):

$$
\begin{gathered}
\min z=\sum_{B \in \mathcal{P}^{k}(N)} m(B) \\
\text { s.t. } \sum_{B \subseteq A, B \in \mathcal{P}^{k}(N)} m(B) \geq \nu(A), \quad \forall A \subseteq N .
\end{gathered}
$$


Notice that (P1) has always a finite optimal solution, since $z$ is bounded below by $\nu(N)$. Take any $\nu^{*} \in \mathcal{C}^{k}(\nu)$, with $m^{*}$ its Möbius transform. Then $m^{*}$ is a feasible solution of (P1), and $z^{*}=\nu(N)$. Hence $m^{*}$ is an optimal solution of (P1). Conversely, suppose that (P1) has an optimal solution $m^{*}$ such that $z^{*}=\nu(N)$. Then clearly $m^{*} \in \mathcal{C}^{k}(\nu)$. Hence, we have shown the following.

Theorem 1. Consider $\nu \in \mathcal{G}(N)$. Then $\nu$ has a nonempty $k$-additive core if and only if the optimal value of the objective function of $(P 1)$ is $z^{*}=\nu(N)$.

Let us consider the dual program of (P1), which we shall call (D1):

$$
\begin{aligned}
& \max q=\sum_{S \subseteq N} \nu(S) y_{S} \\
& \text { s.t. }\left\{\begin{aligned}
\sum_{S \mid A \subseteq S \subseteq N} y_{S} & =1, \quad \forall A \in \mathcal{P}^{k}(N), A \neq \emptyset \\
y_{S} & \geq 0, \quad S \subseteq N .
\end{aligned}\right.
\end{aligned}
$$

By the strong duality theorem, we deduce that a necessary and sufficient condition such that the $k$-additive core is nonempty is that the optimal solution $y^{*}$ of (D1) is such that $q^{*}=\nu(N)$. Hence, we are lead to the following definition and result.

Definition 16. We say that $\mathcal{C} \subseteq \mathcal{P}(N)$ is a $k$-balanced family if there exist positive constants $y_{S}, S \in \mathcal{C}$ such that

$$
\sum_{S \in \mathcal{C} \mid S \supseteq A} y_{S}=1, \quad \forall A \in \mathcal{P}^{k}(N), A \neq \emptyset .
$$

We call $y=\left\{y_{S}\right\}_{S \in \mathcal{C}}$ a $k$-balanced vector for $\mathcal{C}$, whose components are called $k$-balanced coefficients.

Theorem 1 can be then rewritten as follows.

Proposition 4. Consider $\nu \in \mathcal{G}(N)$. Then $\nu$ has a nonempty $k$-additive core if and only if every $k$-balanced family $\mathcal{C}$ with $k$-balanced vector y satisfies:

$$
\sum_{S \subseteq N} \nu(S) y_{S} \leq \nu(N)
$$

The following result shows that there is only one $k$-balanced family, as soon as $k>1$. 
Proposition 5. Let $1<k \leq n$. Then there is a unique $k$-balanced family, which is $\{N\}$, with balanced vector $y_{N}=1$.

Proof: We prove the result first for $k=2$. A first fact is that clearly $\{N\}$ is always a k-balanced collection with balanced vector $y_{N}=1$.

Take $\mathcal{C}$ any 2-balanced family on $N$, with vector $\left\{y_{S}\right\}_{S \in \mathcal{C}}$, and extend the vector $y$ on $\mathbb{R}^{2^{n}-1}$ by putting $y_{S}:=0$ for those $S \notin \mathcal{C}, S \neq \emptyset$. Since $y$ is a 2 -balanced vector, for $A:=\{1\}$ and $A:=\{1,2\}$, we have:

$$
\begin{aligned}
& 1=\sum_{S \in \mathcal{C} \mid\{1\} \subseteq S} y_{S}=\sum_{S \subseteq N \mid\{1\} \subseteq S} y_{S} \\
& 1=\sum_{S \in \mathcal{C} \mid\{1,2\} \subseteq S} y_{S}=\sum_{S \subseteq N \mid\{1,2\} \subseteq S} y_{S} .
\end{aligned}
$$

Substituting (3) into (2) gives

$$
0=\sum_{S \subseteq N \backslash\{2\} \mid 1 \in S} y_{S} .
$$

Since $\{1\}$ and $\{1,2\}$ are arbitrary and $y_{S} \geq 0$ for all $S \subseteq N$, we deduce that $y_{S}=0$ for all $S \in \mathcal{C}, S \neq N$, and $y_{N}=1$. Hence $\mathcal{C}=\{N\}$ is the only possibility.

Now, observe that any $k$-balanced family for $k \geq 2$ is also a 2-balanced family. It follows that for any $k \geq 2$, the only $k$-balanced family is $\{N\}$.

Combining Propositions 4 and 5, we deduce the following.

Corollary 2. For any $k \geq 2$ and any game $\nu \in \mathcal{G}(N), \mathcal{C}^{k}(\nu) \neq \emptyset$.

It is possible to easily construct a 2-additive game $\nu^{*}$ in the 2-additive core of any game $\nu$. Denoting by $m^{*}$ its Möbius transform, put arbitrarily $m^{*}(i)=k_{1}$ and $m^{*}(\{i, j\})=k_{2}$ for any $i, j \in N$. Since $\nu^{*}(N)=\nu(N)$, we get

$$
n k_{1}+\frac{n(n-1)}{2} k_{2}=\nu(N),
$$

hence $k_{2}=2 \frac{\nu(N)-n k_{1}}{n(n-1)}$. Therefore, for any coalition $S$ of $s$ elements:

$$
\nu^{*}(S)=s k_{1}+\frac{s(s-1)}{2} k_{2}=s k_{1}\left(1-\frac{s-1}{n-1}\right)+\frac{s(s-1)}{n(n-1)} \nu(N) .
$$

Thus $\nu^{*}(S)$ is a linear increasing function in $k_{1}$, whence $\nu^{*}(S)$ can be made as large as necessary to obtain dominance. 


\section{$6 \quad k$-balanced monotone families}

We turn now to the problem of finding sufficient and necessary conditions for the nonemptiness of the $k$-additive monotone core of some capacity. Since the approach is similar to the previous section and to [13], we omit proofs.

Consider a capacity $\nu$ on $N$. In this case, if $\nu^{\prime} \in \mathcal{F} \mathcal{M}^{k}(N)$ and $m^{\prime}$ denotes its corresponding Möbius transform, the linear programming problem (P1) changes into (P2):

$$
\begin{aligned}
\min \sum_{B \in \mathcal{P}^{k}(N)} m^{\prime}(B) & =z \\
\text { s.t. } \sum_{B \subseteq A} m^{\prime}(B) & \geq \nu(A), \forall A \subseteq N \\
\sum_{i \in B \subseteq A, B \in \mathcal{P}^{k}(N)} m^{\prime}(B) & \geq 0, \forall i, A, i \in A \subseteq N
\end{aligned}
$$

The first line of constraints is necessary in order to ensure dominance. The second line of constraints in (P2) is needed to ensure monotonicity (Proposition 1).

As for (P1), the following can be shown.

Proposition 6. Given a capacity $\nu$ on $N$, there exists a $k$-additive (at most) capacity $\nu^{*}$ on $N$ dominating $\nu$ if and only if the optimal value of the objective function $z^{*}$ of (P2) is such that $z^{*}=1$.

We consider the dual problem of (P2), that we shall name (D2). It is given by:

$$
\begin{gathered}
\max \sum_{S \subseteq N} \nu(S) y_{S}=q \\
\text { s.t. } \sum_{S \mid A \subseteq S \subseteq N} y_{S}+\sum_{i, S \mid i \in A \subseteq S} y_{i, S}=1, \forall A \in \mathcal{P}^{k}(N), A \neq \emptyset \\
y_{S} \geq 0, y_{i, S} \geq 0, \forall i \in S, \forall S \subseteq N .
\end{gathered}
$$

The variables $y_{S}$ come from dominance constraints. Variables $y_{i, S}$ come from the monotonicity constraints of Equation (6).

Let us denote by $q^{*}$ the optimal value of the objective function of (D2). As before,

$$
z^{*}=1 \Leftrightarrow q^{*}=1
$$


Therefore, in order to find conditions for the $k$-additive core to be nonempty, it suffices to find the conditions for $q^{*}=1$.

We are now in position to introduce the concept of $k$-balanced monotone family:

Definition 17. Consider $\mathcal{Q}$ defined by

$$
\mathcal{Q}:=\{(i, T) \mid i \in T \subseteq N\}
$$

and $\mathcal{C}_{1} \subseteq \mathcal{P}(N), \mathcal{C}_{2} \subseteq \mathcal{Q}$ two collections, at least one of them being nonempty. We say that $\mathcal{C}:=\left(\mathcal{C}_{1}, \mathcal{C}_{2}\right)$ is a $k$-balanced (monotone) family if there exist positive constants $y_{S}, y_{(i, T)}, S \in$ $\mathcal{C}_{1},(i, T) \in \mathcal{C}_{2}$ such that

$$
\sum_{S \in \mathcal{C}_{1} \mid A \subseteq S} y_{S}+\sum_{(i, T) \in \mathcal{C}_{2} \mid i \in A \subseteq T} y_{(i, T)}=1, \forall A \in \mathcal{P}^{k}(N), A \neq \emptyset .
$$

We say then that vector $\vec{y}=\left(y_{S}, y_{(i, T)}\right)_{S \in \mathcal{C}_{1},(i, T) \in \mathcal{C}_{2}}$ is a $k$-balanced (monotone) vector for $\mathcal{C}$ whose components are called $k$-balanced coefficients.

Note that $k$-balanced vectors are the feasible solutions of the dual problem (D2). The elements in $\mathcal{C}_{1}$ correspond to variables in the dominance constraints, while elements in $\mathcal{C}_{2}$ are related to monotonicity conditions.

Example 3. Suppose $|N|=3$, and $k=2$. We give two examples of 2-balanced monotone families:

- $y_{N}=1, y_{A}=0, \forall A \neq N, y_{i, A}=0, \forall i \in A \subseteq N$.

This is the unique 2-balanced family.

- $y_{i}=0.1, y_{\{i, j\}}=0.1, y_{N}=0.1, y_{\{i\},\{i\}}=0.1, y_{\{i\},\{i, j\}}=0.1, y_{i, N}=0.3$, for all $i, j \in N$, $i \neq j$.

This shows that contrarily to the case of games, there is not a single $k$-balanced monotone family.

On the set of all $k$-balanced monotone families, it is possible to define the following operation: 
Definition 18. Given $\mathcal{C}=\left(\mathcal{C}_{1}, \mathcal{C}_{2}\right)$, and $\mathcal{D}=\left(\mathcal{D}_{1}, \mathcal{D}_{2}\right)$ two k-balanced monotone families, we define the union of these families as

$$
\mathcal{C} \cup \mathcal{D}=\left(\mathcal{C}_{1} \cup \mathcal{D}_{1}, \mathcal{C}_{2} \cup \mathcal{D}_{2}\right)
$$

We say that $\mathcal{C}$ is contained in $\mathcal{D}$, denoted by $\mathcal{C} \subseteq \mathcal{D}$, if $\mathcal{C}_{1} \subseteq \mathcal{D}_{1}$ and $\mathcal{C}_{2} \subseteq \mathcal{D}_{2}$.

With these operations, the following can be shown:

Proposition 7. The set of k-balanced monotone families is a sup-semilattice [7], with partial order $\subseteq$ and $\mathcal{C} \vee \mathcal{D}=\mathcal{C} \cup \mathcal{D}$

As a consequence, we have that the union of a finite number of $k$-balanced monotone families is a $k$-balanced monotone family. As $N$ is a finite set, the number of $k$-balanced monotone families is finite. Thus, this implies that the set of all $k$-balanced monotone families is a complete supsemilattice [7].

Proposition 8. Let $\mathcal{C}, \mathcal{D}$ be two k-balanced monotone families satisfying $\mathcal{C} \subset \mathcal{D}$. Then, there exists a $k$-balanced monotone family $\mathcal{B}$ such that $\mathcal{B} \cup \mathcal{C}=\mathcal{D}$ and $\mathcal{B} \neq \mathcal{D}$. Moreover, there exist more than one $k$-balanced monotone vector for $\mathcal{D}$.

Definition 19. We say that a $k$-balanced monotone family $\mathcal{C}$ is minimal if it has no proper $k$-balanced monotone subfamily.

Note that from the definition of minimal $k$-balanced monotone family, these families are atoms in the sup-semilattice of $k$-balanced monotone families [7]. Then, as the set of all $k$-balanced monotone families with the operation $\subseteq$ is a finite sup-semilattice, we have:

Proposition 9. Any k-balanced monotone family can be put as a finite union of minimal $k$ balanced monotone families.

For minimal $k$-balanced monotone families, the following can be proved:

Proposition 10. A $k$-balanced monotone family has a unique $k$-balanced monotone vector if and only if it is minimal. 
Proposition 11. The basic feasible solutions of (P2) are the $k$-balanced monotone vectors of the minimal k-balanced monotone families.

We state now the main result of the section, that gives a necessary and sufficient condition to obtain a $k$-additive capacity dominating another capacity.

Theorem 2. A necessary and sufficient condition for $\nu \in \mathcal{F} \mathcal{M}(N)$ to be a $k$-balanced (at most) capacity is that any minimal $k$-balanced monotone family $\mathcal{C}=\left(\mathcal{C}_{1}, \mathcal{C}_{2}\right)$ with vector $\vec{y}$ satisfies

$$
\sum_{S \in \mathcal{C}_{1}} y_{S} \nu(S) \leq \nu(N) .
$$

\section{Conclusions}

In this paper we have proposed a generalization of the concept of balanced games, called $k$ balanced games. These games are those admitting a dominant $k$-additive game. We have shown that $k$ represents the minimum degree of interaction that we have to permit for the existence of a dominating game. We have derived a representative value for each player based on the pessimistic criterion. From these representative values, we have justified that the value of $k$ can be interpreted as a degree of stability of the game. We think this generalization could shed light on games which are not balanced and might give insight in the theory of Cooperative Games.

We have also looked for necessary and sufficient conditions for a game (resp. a capacity) to be at most $k$-balanced (resp. $k$-balanced monotone). For the case of games, we have shown that the 2-additive core is never empty, which means that any game is either balanced or 2-balanced. The situation for the $k$-additive monotone core is more complex, but any capacity is $k$-balanced monotone for some $1 \leq k \leq n$.

Another problem is determining the $k$-additive core of a given game. This could be interesting in order to give representative values for the players. In the monotone case, this set is a convex polytope, so that it is defined through the vertices. A step in this direction for $k$-additive games can be seen in [11].

We have taken as a basis for our generalization of the core the point of view of coalitional stability. A more fundamental point of view could be to consider non-dominated imputations (see, 
e.g., Owen [13]). In the classical case, the two approaches coincide if the game is superadditive. This could be a very interesting topic to investigate in the future.

\section{Acknowledgements}

This research has been supported in part by grant MTM2007-61193 and CAM-UCM910707. We gratefully thank Mrs. Tong Li, who pointed out the fact that $k$-balanced families for games were reduced to a singleton.

\section{References}

[1] O. Bondareva. Some applications of linear programming to the theory of cooperative games. Problemy Kibernet, (10):119-139, 1963.

[2] A. Chateauneuf and J.-Y. Jaffray. Some characterizations of lower probabilities and other monotone capacities through the use of Möbius inversion. Mathematical Social Sciences, (17):263-283, 1989.

[3] G. Choquet. Theory of capacities. Annales de l'Institut Fourier, (5):131-295, 1953.

[4] A. P. Dempster. Upper and lower probabilities induced by a multivalued mapping. The Annals of Mathematical Statististics, (38):325-339, 1967.

[5] D. Denneberg. Non-additive measures and integral. Kluwer Academic, 1994.

[6] T. Driessen. Cooperative Games. Kluwer Academic, 1988.

[7] P. Dubreil. Algèbre. Vol. 1. Equivalences, opérations, groupes, anneaux, corps. GauthiersVillars, 1946. In French.

[8] M. Grabisch. $k$-order additive discrete fuzzy measures. In Proceedings of 6th International Conference on Information Processing and Management of Uncertainty in Knowledge-Based Systems (IPMU), pages 1345-1350, Granada (Spain), 1996. 
[9] M. Grabisch. Alternative representations of discrete fuzzy measures for decision making. International Journal of Uncertainty, Fuzziness and Knowledge-Based Systems, 5:587-607, 1997.

[10] M. Grabisch. $k$-order additive discrete fuzzy measures and their representation. Fuzzy Sets and Systems, (92):167-189, 1997.

[11] M. Grabisch and P. Miranda. On the vertices of the k-additive core. Discrete Mathematics, to appear.

[12] J. C. Harsanyi. A simplified bargaining model for the n-person cooperative game. Int. Econom. Rev., 4:194-220, 1963.

[13] G. Owen. Game Theory. 3d edition. Academic Press, 1995.

[14] G. C. Rota. On the foundations of combinatorial theory I. Theory of Möbius functions. Zeitschrift für Wahrscheinlichkeitstheorie und Verwandte Gebiete, (2):340-368, 1964.

[15] G. Shafer. A Mathematical Theory of Evidence. Princeton University Press, Princeton, New Jersey, (USA), 1976.

[16] L. S. Shapley. A value for n-person games. In H. W. Kuhn and A. W. Tucker, editors, Contributions to the theory of Games, volume II of Annals of Mathematics Studies, pages 307-317. Princeton University Press, 1953.

[17] L. S. Shapley. Cores of convex games. International Journal of Game Theory, 1:11-26, 1971.

[18] M. Sugeno. Theory of fuzzy integrals and its applications. PhD thesis, Tokyo Institute of Technology, 1974.

[19] P. Walley. Coherent lower (and upper) probabilities. Technical Report 22, U. of Warwick, Coventry, (UK), 1981. 